\title{
Évolutions de la réglementation du travail dans le secteur agricole
}

Maria Katia Garcia Landaburu

\section{(2) OpenEdition}

1 Journals

Édition électronique

URL : https://journals.openedition.org/rdctss/730

DOI : $10.4000 /$ rdctss.730

ISSN : 2262-9815

Éditeur

Centre de droit comparé du travail et de la sécurité sociale

\section{Édition imprimée}

Date de publication : 1 avril 2021

Pagination : 168-171

ISSN : 2117-4350

\section{Référence électronique}

Maria Katia Garcia Landaburu, «Évolutions de la réglementation du travail dans le secteur agricole », Revue de droit comparé du travail et de la sécurité sociale [En ligne], 1 | 2021, mis en ligne le 01 avril 2021, consulté le 03 avril 2022. URL : http://journals.openedition.org/rdctss/730 ; DOI : https:// doi.org/10.4000/rdctss.730

\section{(c) $(7)(9$}

Revue de droit comparé du travail et de la sécurité sociale est mise à disposition selon les termes de la Licence Creative Commons Attribution - Pas d'Utilisation Commerciale - Pas de Modification 4.0 International. 


\title{
MARIA KATIA GARCIA LANDABURU
}

\author{
Université pontificale catholique du Pérou
}

\section{ÉVOLUTIONS DE LA RÉGLEMENTATION DU TRAVAIL DANS LE SECTEUR AGRICOLE}

Le 31 décembre 2020, la loi n³1110 intitulée « Loi sur le régime du travail agraire et les incitations pour le secteur agraire, l'agro-exportation et l'agro-industrie » a été publiée au Journal officiel El Peruano. Avant d'évoquer les principales caractéristiques du nouveau régime du travail, il est important de présenter la réglementation précédente (I), afin de comprendre l'évolution de la législation et le contexte dans lequel la nouvelle loi a été promulguée (II).

\section{I - LES NORMES DE TRAVAIL ANTÉRIEURES EN FAVEUR DE LA PROMOTION DU SECTEUR AGRICOLE}

Le secteur agricole au Pérou est assez hétérogène. II se caractérise par une grande diversité, à la fois des zones où l'activité est exercée (sur la côte, dans les hauts plateaux et dans la jungle), du type de produits cultivés, et des personnes concernées. La loi sur la réforme agraire de 1964 a encouragé l'exploitation des terres directement par leurs propriétaires ou par le biais de coopératives. Cependant, après la période de gouvernement militaire, on a assisté à un retour de la concentration des terres sur la côte, où le climat est favorable, les infrastructures meilleures et l'accès au marché plus grand, tant au niveau national qu'international. Aussi, existe-t-il différents profils d'agriculteurs, allant du petit agriculteur cultivant pour sa propre consommation (voire ayant recours au troc) à la grande entreprise dont les produits sont destinés à l'exportation. C'est pourquoi, d'un point de vue professionnel, il existe des travailleurs indépendants et des travailleurs subordonnés, qui exercent leur activité principalement de manière informelle.

En 1996, par le biais du décret législatif n885 intitulé « Loi de promotion agraire », le gouvernement de Fujimori a déclaré prioritaire l'investissement et le développement du secteur agricole.

Des avantages fiscaux ont alors été mis en place, ainsi que les mesures suivantes en matière de travail et de sécurité sociale :

- l'exonération du paiement par les employeurs des redevances administratives du ministère du Travail et de la Promotion sociale;

- la possibilité de présenter conjointement les contrats temporaires au ministère, à la fin de chaque semestre ;

- l'exonération de la contribution au Fonds national du logement (FONAVI) à la charge du travailleur ;

- et la mise en place d'une assurance maladie avec une cotisation mensuelle de l'employeur équivalente à $4 \%$ du salaire minimum pour chaque travailleur dépendant (cette cotisation était inférieure à celle de $9 \%$ prévue par le régime d'assurance maladie de la sécurité sociale). Cette loi ne prévoyait pas de régime de travail distinct pour les salariés agricoles, qui restaient donc soumis au régime général. 
C'est en 2000 que les nouvelles dispositions relatives à la promotion du secteur agricole ont été adoptées par la loi $n^{\circ} 27360$. Le régime du travail agricole a alors été complété par des règles supplémentaires figurant à l'article 7.2 dont:

- Un salaire journalier (Remuneración Diaria), comprenant la compensation pour un temps de service (CTS ci-après : substitut des indemnités chômage) et les primes versées à l'occasion de la fête nationale et de Noël. Ce salaire journalier était revalorisé proportionnellement au salaire minimum (Remuneración mínima vital). Le salaire journalier initial était de 16 Nuevos Soles; or, si l'on compare ce montant aux 410 Nuevos Soles du salaire minimum, il est évident que le montant de la CTS et des primes était inférieur à celui du régime général du travail.

- 15 jours calendaires de congés payés par année travaillée (contre 30 jours dans le régime général).

- En cas de licenciement arbitraire, une indemnité équivalente à 15 jours de salaire par année travaillée, plafonnée à 180 jours (à l'époque, le régime général prévoyait une indemnité équivalente à un mois de salaire par année travaillée, avec un plafond de 12 mois de salaires).

Autrement dit, pour renforcer le secteur agricole, il a été décidé que les travailleurs bénéficieraient de prestations inférieures à celles du régime général, perçues qui plus est sous forme de rémunération "consolidée ", et non pas en juillet et décembre pour les primes, ni par le biais de dépôts bancaires semestriels pour la CTS.

Cette loi a fait l'objet d'un recours en inconstitutionnalité formé par l'Ordre des avocats de la ville d'Ica (Exp. 00027-2006-PI). En 2007, la Cour constitutionnelle a considéré que l'article 7.2 susmentionné ne violait pas le droit à l'égalité : la loi n²7360 étant une législation spéciale et exceptionnelle, promulguée au regard du contexte, elle n'était donc pas inconstitutionnelle.

Malgré les critiques suscitées par cette décision, l'application du régime s'est poursuivie. Au départ, ce régime devait s'appliquer jusqu'au 31 décembre 2010, mais la loi n²8810 l'a prolongé jusqu'au 31 décembre 2021. En 2019, deux ans avant l'échéance, le décret d'urgence $n^{\circ} 043-2019$ a été publié, portant modification de la loi, réajustant à partir

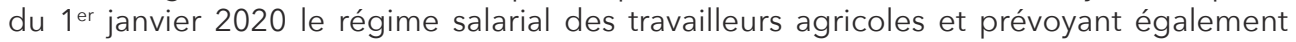
une augmentation progressive de la cotisation de l'employeur au système de santé afin d'améliorer l'accès aux soins des travailleurs (l'objectif était d'atteindre en 2029 les 9\% fixés par le régime général).

L'article 7.2 de la loi a lui aussi été modifié, établissant que le régime du travail agricole répondrait aux conditions suivantes:

Un salaire journalier d'au moins S/ 39,19 Soles. Le salaire journalier comprend : - le salaire de base, qui ne peut être inférieur au salaire minimum ;

- les primes versées à l'occasion de la fête nationale et de Noël, équivalentes à 16,66 \% du salaire de base ;

- et la CTS, équivalente à 9,72\% du salaire de base.

L'ensemble de ces montants devaient être revalorisés proportionnellement à l'augmentation du salaire minimum. Il a été décidé que ces éléments de rémunération seraient enregistrés séparément dans le registre électronique des salariés à des fins de vérification.

a) 30 jours calendaires de congés payés par année travaillée (comme dans le régime général).

b) En cas de licenciement arbitraire, une indemnité équivalente à 45 jours de salaire par année complète travaillée avec un maximum de 360 jours de salaire. Ainsi, l'avantage 
devenait plus élevé que celui prévu dans le régime général car il était calculé en intégrant les primes et la CTS dans le salaire journalier (ce qui n'est pas le cas du régime général). Il convient toutefois de noter que les contrats temporaires sont très répandus dans le secteur agricole et que la résiliation du contrat arrivé à échéance ne donne lieu au paiement d'aucune indemnité.

Les dispositions susmentionnées prévoyaient des avantages économiques similaires à ceux du secteur privé (à l'exception du versement des primes et de la CTS); mais cette loi a été de courte durée. En novembre 2020, presque immédiatement après les manifestations ayant conduit au changement de présidence, de violentes protestations ont éclaté dans les régions où l'activité agricole est intensive. Les mauvais traitements et les conditions de travail déplorables étaient dénoncés alors que le secteur agricole avait enregistré de belles performances sans que cela ne profite aux travailleurs).

Finalement, la loi n²7360 a été abrogée début décembre 2020 par la loi n³1087. Cette dernière cherchait à faire taire les protestations, mais les travailleurs n'étaient toujours pas gagnants : de nouveau soumis au régime général, ils subissaient une réduction temporaire de leurs revenus nets, les primes étant versées en juillet et en décembre et la CTS tous les six mois. En outre, l'abrogation de la loi impliquait la suppression des avantages fiscaux pour le secteur. C'est pourquoi, la loi n³1110, dont il sera question ci-après, a été adoptée juste avant 2021

\section{II - LA LOI SUR LE RÉGIME DU TRAVAIL AGRAIRE ET LES MESURES D'INCITATION EN FAVEUR DU SECTEUR AGRICOLE, DE L'AGRO-EXPORTATION ET DE L'AGRO-INDUSTRIE}

Cette loi contient essentiellement des dispositions régissant le régime du travail. Malgré quelques nouveautés, elle reprend les dispositions de la loi précédente et celles relatives au régime général du travail dont les travailleurs agricoles pourraient alors plus facilement se prévaloir.

\section{A- LA RÉMUNÉRATION}

Trois modalités sont mises en place :

- Un salaire de base, qui ne peut être inférieur au salaire minimum. II permet de fixer le montant des primes légales et la CTS, équivalant respectivement à $16,66 \%$ et $9,72 \%$ du salaire de base.

- Un salaire journalier correspondant au montant de la somme des éléments précités, divisée par 30, à condition que le salarié travaille plus de quatre heures par jour en moyenne. Néanmoins, il a été établi que le travailleur pouvait, à titre facultatif, choisir de percevoir les primes et la CTS dans les conditions prévues par la loi, auquel cas, elles ne seraient plus comprises dans le salaire journalier.

- Une prime spéciale pour les travaux agricoles (ou BETA, pour Bonificación Especial por Trabajo Agrario), équivalente à 30\% du salaire minimum. Cette prime n'est pas considérée comme faisant partie du salaire, et de ce fait, n'est pas prise en compte dans la base de calcul des prestations comme les primes et la CTS.

\section{B- LES DROITS DU TRAVAIL}

Comme indiqué précédemment, les avantages prévus dans la loi précédente ont été maintenus : 30 jours de congés payés et, en cas de licenciement arbitraire, une indemnité équivalente à 45 jours de salaire par année travaillée, avec un maximum de 360 jours de salaire. 
Les règles applicables au régime commun ont également été incorporées : la journée de travail ordinaire ne doit pas dépasser 8 heures par jour ou 48 heures par semaine ; au-delà, les heures travaillées sont considérées comme des heures supplémentaires et doivent être payées avec une majoration d'au moins $25 \%$ pour les deux premières heures et $35 \%$ pour les heures suivantes, sauf si les parties conviennent de compenser le travail effectué en heures supplémentaires par des périodes de repos équivalentes; le travail de nuit (entre 22 heures et 6 heures) doit être rémunéré avec une majoration de 35 \% par rapport au salaire minimum ; les allocations familiales doivent être proportionnelles au nombre de jours travaillés.

En outre, une augmentation progressive de la participation des travailleurs aux bénéfices de l'entreprise a été instaurée : 5\% de 2021 à 2023 ; 7,5\% de 2024 à 2026 et 10\% à partir de 2027.

\section{C- LE DROIT PRÉFÉRENTIEL DE RECRUTEMENT}

Le droit préférentiel de recrutement est prévu sous conditions, dans les cas d'embauche pour de courtes durées, ou pour des périodes supérieures à deux mois sur une année, ou encore en cas d'embauche intermittente, saisonnière ou assimilée par le même employeur, ou en cas d'embauche par des entreprises liées.

\section{D- LES CONDITIONS DE TRAVAIL}

La loi indique que les employeurs doivent garantir à leurs salariés des conditions de travail décentes et sûres, devant être mises en œuvre conformément à la réglementation. Celles-ci doivent nécessairement inclure : le transport des travailleurs vers le lieu de travail, la nourriture, les soins d'urgence, les services sanitaires appropriés et des lieux dédiés, ainsi que la mise en place de mesures sanitaires et d'hygiène adéquates. La loi prévoit également des conditions de travail spéciales pour les femmes salariées et pour les mineurs, en incorporant des dispositions issues du régime général.

\section{E - L'EXERCICE DES DROITS COLLECTIFS}

La nouvelle loi vise à promouvoir le droit à la négociation collective, en particulier au-delà du niveau de l'entreprise, car les travailleurs agricoles et de l'agro-exportation ont des difficultés à exercer ce droit de manière efficace, en raison de la discontinuité et du caractère saisonnier de leurs activités. À cette fin, le ministère du Travail et de la Promotion de l'Emploi (MTPE) est tenu d'élaborer un règlement portant sur la négociation collective ; la loi sur les relations collectives de travail a également été modifiée et comprend désormais l'article 45-A, qui se réfère spécifiquement à ce régime de travail et comprend des dispositions en cas de conflits entre conventions collectives de différents niveaux.

Comme on peut le voir, le renforcement du secteur agricole ne passe plus par une limitation des droits des travailleurs mais par la prise en compte des spécificités inhérentes au secteur dans la réglementation d'un certain nombre de questions, et la mise en place d'avantages supplémentaires. II n'est pas possible d'évaluer l'effet de ces mesures, mais il est clair que le régime précédent a nui aux travailleurs du secteur, qui s'est développé principalement grâce à l'ouverture à d'autres marchés, et à des tarifs qui lui étaient favorables. 